BUCHBESPRECHUNGEN

DOI: $10.1007 / \mathrm{s} 00350-010-2662-9$

\section{Medizin und Haftung. Festschrift für Erwin Deutsch zum 80. Geburtstag.}

Herausgegeben von Hans-Jürgen Ahrens, Christian von Bar, Gerfried Fischer, Andreas Spickhoff und Jochen Taupitz. Springer-Verlag, Berlin, Heidelberg 2009, XIV u. 1104 S., geb., $€ 159,95$

Der vielfach ausgezeichnete Jubilar gehört zu den produktivsten Rechtswissenschaftlern. Auch in seinem achten Lebensjahrzehnt hat er eine bewundernswerte Reihe selbständiger Schriften, Aufsätze, Urteilsanmerkungen und Rezensionen publiziert, welche die vorliegende neuerliche Festschrift im Anschluß an diejenige zum Siebzigsten von 1999 verzeichnet (S. 1097-1104). Das hier anzuzeigende, überaus reichhaltige Gemeinschaftswerk spiegelt mit seinen nicht weniger als siebenundsechzig Beiträgen die Arbeitsfelder von Erwin Deutsch: Dreiundvierzig der alphabetisch nach Autorennamen angeordneten Aufsätze gelten dem inzwischen weit gewordenen Thema Medizin und Recht. Dabei bildet die Forschung am Menschen mit der Arzneimittelprüfung und ihren Annexen einen Schwerpunkt. Zum Haftungs- und Versicherungsrecht sind achtzehn Autoren versammelt, und abschließend befassen sich sechs mit Grundfragen des Zivilrechts. Unter den Beiträgen finden sich, dem Oeuvre des Jubilars angemessen, Arbeiten interdisziplinären, europäischen und international-rechtsvergleichenden Inhalts. Den verschiedenartigen, durchweg aktuellen und weiterführenden Studien geht ein liebenswürdiges, persönlich gehaltenes Geleitwort von Andreas Spickhoff voraus.

Die Opulenz des weit über tausend Seiten starken Buches setzt den Rezensenten in die Verlegenheit, nur Teile des Inhalts ansprechen zu können und dabei auch noch - angesichts der allgemein hohen Qualität - fast willkürlich verfahren zu müssen. Der Leser findet grundlegende Aufsätze allgemeineren Zuschnitts: Thorsten Kingreen bringt Medizinrecht und Gesundheitsrecht auf den Begriff und faßt letzteres als ,,die Gesamtheit der öffentlich-rechtlichen Normen über die krankenversicherungsrechtliche Stellung der Versicherten, die Organisation und Finanzierung der Krankenkassen und den berufsund sozialversicherungsrechtlichen Status der in der Gesundheitsversorgung tätigen Personen und Einrichtungen“"

Udo Steiner befaßt sich mit der unter dem „Bleigewicht“ der Gesetzlichen Krankenversicherung und den Notwendigkeiten ihrer Finanzierung leidenden ärztlichen Freiberuflichkeit. Anschaulich beleuchten Heinz Pichlmaier und Hans Friedrich Kienzle Rechte und

Prof. Dr. iur. Dr. h.c. Adolf Laufs,

Heidelberg, Deutschland
Pflichten des Arztes. Für partnerschaftlichen Konsens in der Intensivmedizin treten - auch sie erfahrene Mediziner - Hilmar Burchardi und Friedemann Nauck ein mit konkreten Ratschlägen.

Auch aus dem alten Kernstück des Arztrechts, der Haftpflicht, seien einige Beiträge zu den Grundlagen genannt: Die Eigenheiten des Arzthaftpflichtprozesses skizziert der erfahrene Vorsitzende Richter Gerhard H. Schlund. Der Beweiserleichterung nach groben und einfachen Arztfehlern widmet sich kritisch und weiterführend Ulrich Foerste. Der renommierte Bundesrichter und Fachschriftsteller Erich Steffen bedenkt die evidenz-basierte Medizin und betont zu Recht, dass Behandeln ,am Kranken mit seinen höchst individuellen Befindlichkeiten und Potentialen für das Gesundwerden" zu geschehen habe. In der Neulandmedizin bildet die Nutzen/Risiko-Relation einen Hauptpunkt. Instruktiv erörtert dazu Dieter Hart Abwägung, Aufklärung und Verfahren für klinische Arzneimittelprüfungen. Dem heiklen Probandenschutz wendet sich Volker Lipp behutsam zu: die Notwendigkeit der Forschung auch an Kindern und einwilligungsunfähigen Erwachsenen erkennend, ohne deren gesteigerte Schutzbedürftigkeit zu ignorieren. Einen akzeptablen Kompromiß empfiehlt auch Gunnar Duttoe, der das strikte Verbot der Arzneimittelprüfung an zwangsweise Untergebrachten kritisch überprüft. In seinen bedenkenswerten Erwägungen zu Überwachung und Prüfung der Transplantationsmedizin wirbt Hans Lilie für eine strikte berufsrechtlich orientierte Kontrolle, die wegen der damit verbundenen Sachkunde eine wesentlich höhere Effektivität verspreche. Über Aufgabe und Legitimation der Bundesärztekammer im Blick auf die Standardisierung in der Transplantationsmedizin schreibt Henning Rosenau in trefflichem Verständnis der ärztlichen Aufgabe. $\mathrm{Zu}$ den großen, sich nicht erschöpfenden Dauerthemen gehören der ärztliche Behandlungsabbruch und die Änderung der Therapieziele am Lebensende, neuerlich über Fachgrenzen hinweg gemeinsam bedacht von Gerhard A. Müller und Jan Knöbl, sowie die inzwischen gesetzlich geregelte Patientenverfügung, die der Altmeister Wilhelm Uhlenbruck in ihrer Entwicklung erörtert; wobei er am Ende begründetermaßen „mehr Palliativ- und Hospizeinrichtungen sowie eine ausreichende Schmerztherapie durch Hausärzte" fordert. Hans-Ludwig Schreiber hingegen kommt zu dem wohlbegründeten Schluß, die Patientenverfügung stelle ein begrenztes Hilfsmittel, aber keine sachliche Lösung der Sterbehilfefrage dar.

Aus dem französischen Recht entnehmen begrüßenswerterweise Anregungen für das deutsche Christian Katzenmeier und Jonas Knetsch, um die „Kind als Schaden“-Problematik zu entlasten und gebotene Hilfe zu gewährleisten durch den Ausbau öffentlicher Leistungen. Die spezielleren Arbeiten zum Medizinrecht besetzen ein weites Feld bis hin zu der so gelehrten wie reizvollen Studie Eva Schumanns über Ärzte und Kranke in den Volksrechten des Frühmittelalters.

Auch die beiden anderen Teile des Bandes bieten anspruchsvolle allgemeinere wie speziellere Stücke, die das Werk für jede Bibliothek unverzichtbar machen. 